\title{
Center-of-mass motion and cross-channel coupling in time-dependent Hartree-Fock theory
}

\author{
A S Umar and V E Oberacker \\ Department of Physics and Astronomy, Vanderbilt University, Nashville, Tennessee \\ 37235 , USA \\ E-mail: umar@compsci.cas.vanderbilt.edu
}

\begin{abstract}
We provide a discussion of issues related to the center-of-mass motion and cross-channel coupling in applications of the time-dependent Hartree-Fock (TDHF) theory to heavy-ion collisions. We find that the entrance channel dynamics of a heavyion collision as described by TDHF does not seem to be significantly influenced by these effects, whereas the long-time evolution may be less reliable.

PACS numbers: 21.60.Jz,24.10.Cn
\end{abstract}

Submitted to: J. Phys. G: Nucl. Phys.

\section{Introduction}

It is generally acknowledged that the time-dependent Hartree-Fock (TDHF) theory provides a useful foundation for a fully microscopic many-body theory of low-energy heavy-ion reactions [1]. The success of the TDHF method is predicated on the expectation that the Pauli principle plays an important role in simultaneously building up a time-dependent mean-field and suppressing the propagation of the strong $N-N$ interaction terms. In recent years there has been a significant progress in the study of heavy-ion collisions using the TDHF theory [2, 3, 4]. This progress is partially due to the available computational power which allows calculations without resorting to simplifying but somewhat unphysical assumptions used in the past, as well as to the considerable improvements made in the parametrization of effective interactions [5].

One of the issues faced in the reduction of the finite many-body problem to be described in terms of single-particle degrees of freedom and a mean-field is the centerof-mass (c.m.) motion. This is due to the fact that the resulting wavefunction, say a Slater determinant, does not factorize into a product of one single center-of-mass wavefunction and a wavefunction of the internal coordinates [6]. Thus the calculated energies include a contribution from the center-of-mass motion in a complicated way. Another way of describing the problem is that the coordinates of the nucleons in the intrinsic 
wavefunction, $\chi\left(\mathbf{r}_{1}, \mathbf{r}_{2}, \ldots, \mathbf{r}_{A}\right)$, should be defined with respect to a fixed origin, which means the coordinates are not independent and satisfy $\sum_{i=1}^{A} \mathbf{r}_{i}=0$.

The second problem faced in TDHF has to do with exit channel properties for collisions where more than one final fragment exists, e.g. deep-inelastic collisions. In this case, although we may have two well separated fragments, the final Slater determinant cannot be transformed to a block diagonal form thus making the identification of asymptotic channels unclear. This is known as the cross-channel coupling problem.

In the sections below we shall discuss the ramifications of these issues to the interpretation of the results obtained from TDHF calculations. In particular we shall discuss each problem in a more detailed fashion and show the magnitude of the effect and try to establish the conditions under which these issues may have a minimal effect on the calculated observables.

\section{C.M. Motion and TDHF}

For many-body wavefunctions obtained using variational calculations there is no well defined prescription for eliminating the center-of-mass coordinate. Most of the suggested methods can be described by the general expression given by Lipkin [7]

$$
\Psi\left(\mathbf{r}_{1}^{\prime}, \ldots, \mathbf{r}_{A-1}^{\prime}\right)=\int g(\mathbf{R}) \Phi\left(\mathbf{r}_{1}, \ldots, \mathbf{r}_{A}\right) d \mathbf{R},
$$

where the function $\Phi$ is the general wavefunction depending on the coordinates of $A$ nucleons, the function $g(\mathbf{R})$ is the weight function for integrating over the center-ofmass coordinate $\mathbf{R}$, and the result is the wavefunction $\Psi$ depending on the $3(A-1)$ internal coordinates. An equivalent expression in momentum space is given in Ref. [8]. One common derivative of the above is to project out the $\mathbf{P}=0$ part of the many-body wavefunction. The proper way of choosing the weight function, $g(\mathbf{R})$, is such that the expectation value of the internal Hamiltonian (i.e. the Hamiltonian after the removal of the center-of-mass dependence) for the wavefunction (11) is a minimum. A proof for the above statement is given in Section 4 of Ref. 9]. In practice this turns out to be a very difficult task. Alternatively, one can specify the functional form for $g(\mathbf{R})$ but include variational parameters, similar to the Lagrange parameters for constraints, in the definition. The variational minimization then would also include these parameters thus giving the best $g(\mathbf{R})$ for the chosen functional form [9]. While these methods may be useful for the calculation of static properties, a generalization to dynamical calculations poses serious technical problems and therefore are not used in practice. As discussed below, TDHF initial wavefunctions actually need the center-of-mass wavefunction to allow for boosts.

If one is primarily interested in energies an alternate procedure is the subtraction

of the center-of-mass energy from the total kinetic energy of the system. If this is done during the minimization procedure one may effectively achieve the above task. Formally, 
this can be written as:

$$
\begin{aligned}
\hat{K}-\hat{K}_{c . m .} & =\sum_{i=1}^{A} \frac{\mathbf{p}_{i}^{2}}{2 m}-\frac{\left(\sum_{i=1}^{A} \mathbf{p}_{i}\right)^{2}}{2 m A} \\
& =\frac{1}{2 m}\left[1-\frac{1}{A}\right] \sum_{i=1}^{A} \mathbf{p}_{i}^{2}-\frac{1}{2 m A} \sum_{i \neq j} \mathbf{p}_{i} \cdot \mathbf{p}_{j} .
\end{aligned}
$$

The last part of this expression is numerically difficult as it includes double integrals and is usually neglected. Consequently, most Skyrme forces use the simple correction to the kinetic energy given in the first term. There are various Skyrme fits that include the quadratic term as well, such as the SLy6 parametrization [5]. The general statements made about this correction is that it is not very sensitive to deformation but it can be substantial in magnitude. A comprehensive study of the influence of the centerof-mass correction in mean-field studies is given in Ref. [10]. This problem has been recently raised in Ref. [11] in the context of calculating fission barriers, where a corrective procedure was suggested.

In TDHF, the static Hartree-Fock Slater determinant is boosted by multiplying each Slater determinant with an exponential phase factor

$$
\Phi_{j} \rightarrow \exp \left(i \mathbf{k}_{j} \cdot \mathbf{R}_{j}\right) \Phi_{j},
$$

where $\Phi_{j}$ is the HF wavefunction for nucleus $j, \mathbf{R}_{j}$ is the corresponding center of mass coordinate

$$
\mathbf{R}_{j}=\frac{1}{A_{j}} \sum_{i=1}^{A_{j}} \mathbf{r}_{i},
$$

and $\mathbf{k}_{j}$ is the boost momentum chosen to be tangent to the Coulomb trajectory at an initial separation distance. The target and projectile are placed to be in the center-ofmass frame for the entire system and satisfy

$$
A_{T} \mathbf{R}_{T}+A_{P} \mathbf{R}_{P}=0 .
$$

Thus, the total momentum for the system has expectation value zero, and the relative momentum is correctly given by $\mathbf{k}_{T}-\mathbf{k}_{P}$. Here, the assumption is that the physics of the collision is determined by the relative momenta. This is one of the reasons why we expect the contributions coming from the wave packets contained in the initial Slater determinants to a large extent cancel out. However, as we discuss below, there are other reasons as to why projecting out the center-of-mass part of the wavefunctions is not appropriate in TDHF calculations.

In TDHF the initial boosted states are interpreted as wave packets because the wave packet is already contained in the static Slater determinants [12, 13]. The result of multiplication by the phase factor given above is to shift the center of momentum of the wave packet by the vector $\mathbf{k}$. However, there are fundamental differences between a boosted TDHF solution and a Schrödinger wave packet. Since we are using a single Slater determinant we are not free to change the wave packet nature of the 


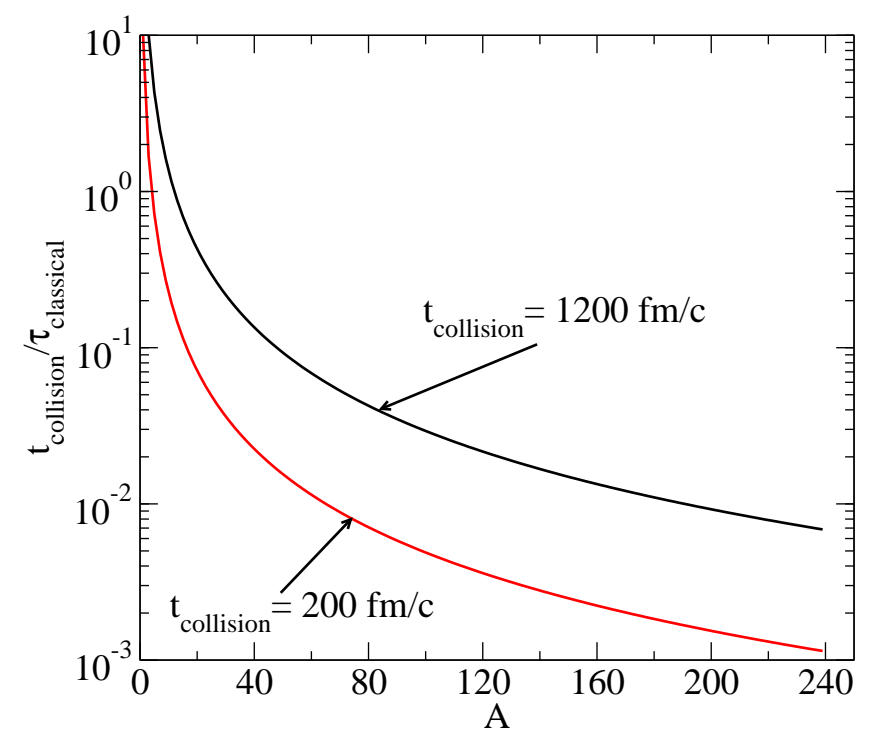

Figure 1. The ratio $t_{\text {collision }} / \tau_{\text {classical }}$ plotted as a function of mass number $A$, for two collision times.

solution. The two are coupled via the minimization process and this goes beyond just the contribution/correction to static energies. This coupling results in a non-spreading wave packet for the TDHF evolution [1]. It can be shown that the TDHF wave packet is identical to the Schrödinger wave packet when the terms causing the spreading in the Schrödinger wave packet are neglected [14, 12]. The condition that the wave packet behave completely classically over the time scale of the collision requires [14, 15] (i.e. the spreading of the wave packet never catches up with its kinematical motion)

$$
\frac{t_{\text {collision }}}{\tau_{\text {classical }}} \ll 1 \quad, \quad \tau_{\text {classical }}=\left[\frac{\hbar}{M \sigma^{2}}\right]^{-1},
$$

where $\sigma$ is the root-mean-square width of the Gaussian wave packet representing the nucleus, and $M$ is the total mass of the nucleus. If we take the initial wave packet to be characterized by the size of the nucleus we can write

$$
\tau_{\text {classical }}=\frac{2 m c^{2} r_{0}^{2} A^{5 / 3}}{\hbar c} \approx 19 A^{5 / 3} \mathrm{fm} / c
$$

Corresponding spreads in c.m. momentum and energy are given by [1]

$$
\Delta p_{c . m .}>\frac{\hbar}{2 r_{0} A^{1 / 3}},
$$

with the corresponding spread in beam-energy

$$
\frac{\Delta E}{E}=\frac{\hbar}{\left(2 m E r_{0} A^{4 / 3}\right)^{1 / 2}},
$$

where $E$ is the beam energy per particle. For light ions this spread could be as much as $10 \%$ but reduces for heavier systems.

In Fig. 1 we plot the dependence of the ratio in Eq. (15) on the mass number for two different reaction times. The longer time scale of $1200 \mathrm{fm} / \mathrm{c}$ refers to the whole 
reaction, while the shorter time scale of $200 \mathrm{fm} / \mathrm{c}$ describes the initial evolution until the nuclei begin to overlap. We see that for heavy systems the ratio is small and the TDHF wave packet may be considered as the classical limit of a Schrödinger wave packet. On the other hand, for long reaction times and light systems the correspondence does not seem to be very good. However, this argument may be flawed since one can take the position that we are only interested in the evolution of the wave packet until the two nuclei begin to have substantial overlap [12]. In this case the collision times are much smaller thus resulting in a smaller ratio for most mass numbers, as shown in Fig. 1. In this context one can interpret the TDHF results from a classical perspective, which is the correct limit for a non-spreading wave packet. Consequently, center-of-mass corrections are not appropriate in TDHF calculations and have always been neglected. The effect of using Skyrme forces fitted with the simple c.m. correction term without this correction in TDHF will be discussed further in the next section. Implications

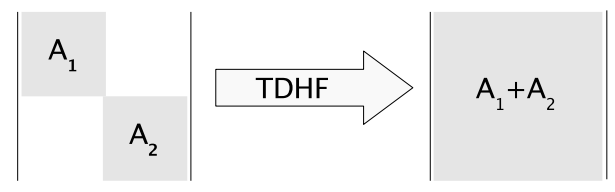

Figure 2. Schematic illustration of the initial and final many-body states. The initial state is block diagonal whereas the final state is a full Slater determinant.

of the above uncertainties depend on the details of the collision process under study. Reactions that are predominantly determined by the entrance channel, such as the initial formation of the compound system in fusion, or the calculation of fusion barriers using the density-constrained TDHF (DC-TDHF) method [16] are expected to be most reliable. On the other hand longer time processes such as deep-inelastic scattering will be prone to higher uncertainties. For example, in calculating Wilczynski plots (doubledifferential cross-section with respect to scattering angle and energy) for deep-inelastic collisions we may expect a spread in energy, as much as $10 \mathrm{MeV}$ for light systems and a spread in impact parameter of order $1 \mathrm{fm}$.

\section{Cross-Channel Coupling}

The so-called cross-channel coupling describes the problem that in deep-inelastic TDHF collisions the final fragments remain entangled. This complicates the identification of exit channel fragments as distinct nuclear systems [17]. In TDHF, the entrance channel asymptotic conditions are set up correctly as discussed in the previous section. In practice, the initial nuclei are calculated using the static Hartree-Fock theory without the center-of-mass correction. The resulting Slater determinants for each nucleus comprise the larger Slater determinant describing the colliding system during the TDHF evolution, as depicted in Fig. 2, Nuclei are assumed to move on a pure Coulomb trajectory until they reach the initial separation between the nuclear centers used in the 
TDHF evolution. Using the Coulomb trajectory we compute the relative kinetic energy at this separation and the associated translational momenta for each nucleus in the center-of-mass frame. The nuclei are then boosted by multiplying each HF determinant by an exponential phase as in Eq. (2) so that the relative momentum is the correct one calculated for the Coulomb collision. The Galilean invariance of the TDHF equations with the full Skyrme force results in the evolution of the system without spreading and the conservation of the total energy for the system. In other words, a translating Slater determinant does not dissipate its kinetic energy. In TDHF, the many-body state remains a Slater determinant at all times. The final state is a filled determinant, even in the case of two well separated fragments (does not go back to block-diagonal form). This phenomenon is commonly known as the cross-channel coupling and indicates that it is not possible to identify the well separated fragments as distinct nuclei since each single particle state will have components distributed everywhere in the numerical box. In this sense it is only possible to extract inclusive (averaged over all states) information from these calculations.

In order to test the validity of the initial TDHF setup and the use of the Skyrme interaction without the c.m. correction term used in the parametrization we utilize the density constrained TDHF method [18, 19]. In this method we constrain the instantaneous TDHF density and minimize the energy. This in effect corresponds to the extraction of the internal excitation energy from the evolving system thus tracing the dynamical trajectory on the multi-dimensional static energy surface of the composite nuclear system. In Ref. [16] we have shown that the ion-ion potential barrier could simply be calculated by subtracting the binding energies of the two nuclei (calculated without the c.m. correction term) as $V(R)=E_{D C}-E_{A_{1}}-E_{A_{2}}$. This in principle tests both the accuracy of the entrance channel evolution and the energy calculation since for relatively large distances the result must agree with the point Coulomb potential (for spherical systems). In Fig. 3a we show the results calculated for the head-on (zero impact parameter) collision of the ${ }^{16} \mathrm{O}+{ }^{16} \mathrm{O}$ system at $E_{\text {c.m. }}=34 \mathrm{MeV}$. Also shown is the double folding result for the same system, using densities obtained from electronscattering and the M3Y effective NN interaction. In Fig. 3b we show the barriers calculated for ${ }^{40} \mathrm{Ca}+{ }^{90,96} \mathrm{Zr}$ systems. Comparison with the point Coulomb expression as shown in Fig. 3b is excellent with differences on the order of $50 \mathrm{keV}$, until nuclear effects come into play. Thus, we can conclude that not including the c.m. correction energy in TDHF calculations does not seem to alter the results when energy differences are considered. Furthermore, we have also performed the same calculations by changing the collision energy with no appreciable change in results indicating that the entrance channel TDHF dynamics is not prone to uncertainties present in the long-time evolution case. 

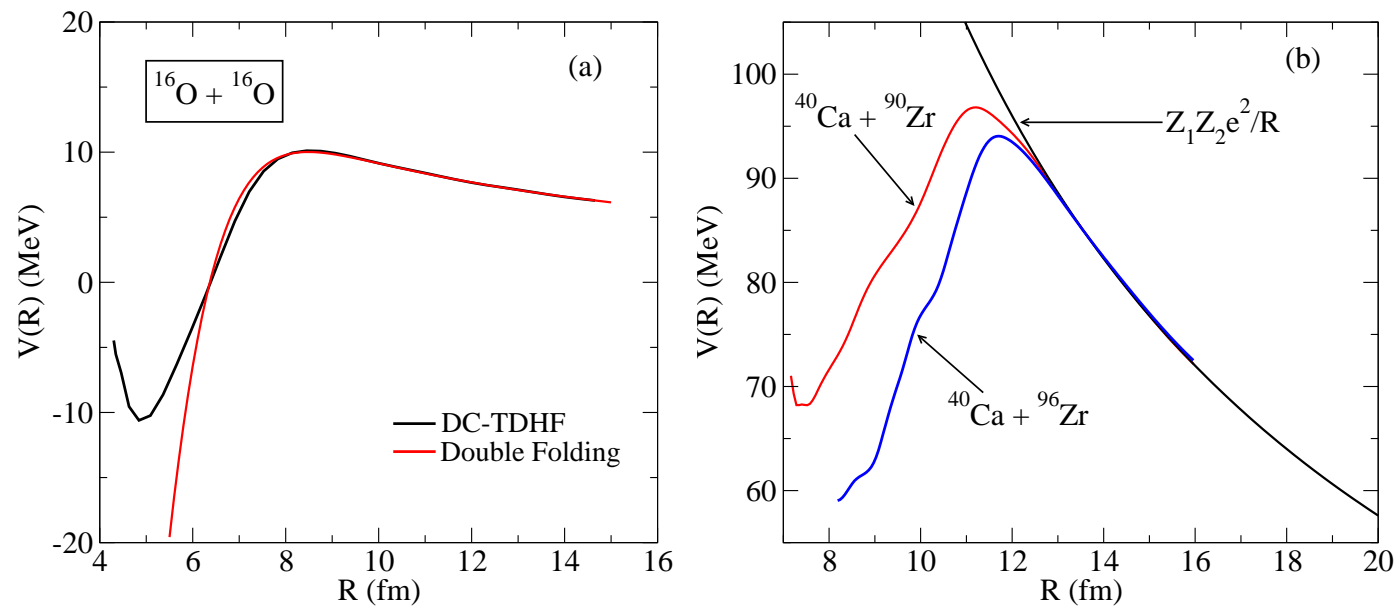

Figure 3. (a) Comparison of the DC-TDHF heavy-ion potential for ${ }^{16} \mathrm{O}+{ }^{16} \mathrm{O}$ to the empirical double-folding potential. (b) DC-TDHF heavy-ion potentials for ${ }^{40} \mathrm{Ca}+{ }^{90,96} \mathrm{Zr}$ and comparison to the point Coulomb potential.

\section{Conclusions}

TDHF calculations in general do not use the center-of-mass correction terms present in most parametrizations of the Skyrme interaction. In this paper, we discuss this issue along with the problem of cross-channel coupling. We also provide estimates and numerical tests to understand the impact of these assumptions. This work, together with the recent investigations provided in Ref. [20], where the authors discuss the conservation of angular momentum in TDHF theory, find that the entrance channel dynamics of a heavy-ion collision is expected to be well described by the TDHF time evolution. Also, when energy differences are taken (e.g. in the calculation of heavy-ion potentials) the omission of the center-of-mass correction terms do not seem to alter the results. Uncertainties are expected for long-time evolution resulting in well separated final fragments.

\section{Acknowledgments}

This work has been supported by the U.S. Department of Energy under grant No. DE-FG02-96ER40963 with Vanderbilt University

\section{References}

[1] Negele J W 1982 Rev. Mod. Phys. 54913

[2] Umar A S and Oberacker V E 2006 Phys. Rev. C 73054607

[3] Maruhn J A, Reinhard P-G, Stevenson P D, Stone J R and Strayer M R 2006 Phys. Rev. C 74 027601

[4] Simenel C, Chomaz P and France G de 2001 Phys. Rev. Lett. 862971

[5] Chabanat E, Bonche P, Haensel P, Meyer J and Schaeffer R 1998 Nucl. Phys. A635 231; Nucl. Phys. A643 441 
[6] deShalit Amos and Feshbach Herman 1974 Theoretical Nuclear Physics, (Wiley, New York)

[7] Lipkin H J 1958 Phys. Rev. 1101395

[8] Peierls R E and Thouless D J 1962 Nucl. Phys. 38154

[9] Bouten M, Bouten M C and Cornelissens T 1985 J. Phys. G 11231

[10] Bender M, Rutz R, Reinhard P-G and Maruhn J A 2000 Eur. Phys. J. A7 467

[11] Skalski J 2007 Phys. Rev. C 76044603

[12] Flocard H, Koonin S E and Weiss M S 1978 Phys. Rev. C 171682

[13] Irvine J M 1980 J. Phys. G 6 L175

[14] Umar A S, Strayer M R, Ernst D J and Sandhya Devi K R 1984 Phys. Rev. C 301934

[15] Goldberger M L and Watson K M 1975 Collision Theory, (Robert E. Krieger Publishing Company, Inc., NY) p.64.

[16] Umar A S and Oberacker V E 2006 Phys. Rev. C 74 021601(R)

[17] Koonin S E, Davies K T R, Maruhn-Rezwani V, Feldmeier H, Krieger S J and Negele J W 1977 Phys. Rev. C 151359

[18] Cusson R Y, Reinhard P-G, Strayer M R, Maruhn J A and Greiner W 1985 Z. Phys. A 320475

[19] Umar A S, Strayer M R, Cusson R Y, Reinhard P-G and Bromley D A 1985 Phys. Rev. C 32172

[20] Guo Lu, Maruhn J A, Reinhard P-G and Hashimoto Y 2008 Phys. Rev. C 77 041301(R) 